\title{
A Second Order Sliding Power Control \& Resonant Filtering Approach to Mitigate Grid Unbalance Effects on a DOIG Wind Energy Based System
}

\author{
F. Valenciaga ${ }^{1}$, R. D. Fernández ${ }^{2}$ and F. Inthamoussou ${ }^{1}$ \\ ${ }^{1}$ Grupo de Control Aplicado (GCA), LEICI - Departamento de Electrotecnia, Fac. de Ingeniería \\ Universidad Nacional de La Plata - CONICET \\ 48 y $116 \mathrm{~s} / \mathrm{n}$, La Plata (Argentina) \\ Phone/Fax number: +0054 221 4259306, e-mail: fval@ing.unlp.edu.ar, intha@ing.unlp.edu.ar \\ ${ }^{2}$ Fac. de Ingeniería, Universidad Nacional de la Patagonia San Juan Bosco (UNPSJB) - CONICET \\ Ciudad Universitaria, Ruta Provincial $\mathrm{N}^{\circ} 1 \mathrm{~s} / \mathrm{n}$, Comodoro Rivadavia (Argentina) \\ Phone/Fax number: +0054 295 4550836, e-mail: dfernandez@unpata.edu.ar
}

\begin{abstract}
This paper presents a multivariable power control design applied to a DFIG Wind Energy Conversion based System (WECS). The multiple control objectives pursued in this work include not only tracking temporal profiles of active and reactive power grid injection but also regulate some important internal variables like the DC bus voltage. To fulfil these targets a multivariable control scheme is developed following second order sliding mode techniques. This theoretical framework allows synthesizing controllers with attractive features like finite time convergence, robustness against external perturbations and unmodeled dynamics, simple implementation, etc. To endorse the control system with grid support features under voltage unbalances the multivariable SOSM controller is combined with a set of resonant filters. This mixed scheme allows to substantially reduce the oscillations of simple and double grid frequency on the electric torque and the power injected into the grid. The overall performance of this control proposal is evaluated using representative simulations.
\end{abstract}

\section{Key words}

Wind Energy Conversion Systems, Grid Unbalances, Multivariable Control Systems

\section{Introduction}

Nowadays, one of the most required features for grid connected wind energy conversion systems (WECS) is that they can operate like conventional generation systems. In this sense the relatively new Grid Connection Codes for wind turbines specify how WECS must overcome without disconnection different kind of usual grid faults like voltage sags, voltage unbalances, frequency drifts, etc. Additionally, they recommend that WECS should be able to provide ancillary services to sustain the grid stability during (and after) faults [1]. In this context, the general control objectives should be directed to regulate the active and reactive power that WECS inject into the utility grid without putting aside the control of some important internal variables that provide for a fast recuperation after faults [2].

Statistically, one of the main grid faults is the presence of unbalanced voltages. This kind of anomaly causes important double grid frequency oscillations on the active and reactive output powers and also in the electromagnetic torque. These strong oscillations are unacceptable from the stability point of view because they introduce important current perturbations into the electric grid and also because produce a dangerous level of stress on the mechanical structure [3][4].

During the last years different control alternatives have been reported trying to tackle the problem of mitigate the effects produced by this kind of anomaly. Most of them are based on sequence decomposition of the grid voltage $[4][5][6][7]$. However, depending on how this sequence decomposition is calculated delays and amplitude/phase errors can be introduced into the loop affecting the dynamic response and control stability [3].

In order to avoid time consuming calculus and its associated errors, this work explores a control scheme to directly drive the power injected into the utility grid. This proposal is mainly based on a multivariable second order sliding mode controller. This technique permits synthesize simple and robust controllers for nonlinear dynamic systems in the presence of bounded unmodeled dynamic and external perturbations with additional attractive characteristics [8][9]. Regretfully, the robustness features of this kind of controllers are not enough to deal with perturbations like the ones produced by voltage unbalances. To endorse the system with this capability, a set of resonant filters is included into the control scheme. This action line proposed by Zhou et al. in [3] gives the system the ability to efficiently reject 
most of the oscillations present on the torque and electric powers, producing at the same time, a simpler control scheme than the one involving sequence decomposition.

\section{Dynamic Model}

The structure of the WECS treated in this work is shown in Fig. 1. It is composed by a DFIG with the stator windings directly linked to the utility grid and the rotor ones connected to the same electric network using a fractional power back to back (B2B) converter. From the mechanical point of view this generator is linked to the wind turbine through a gear box of relationship $1: K_{g b}$.

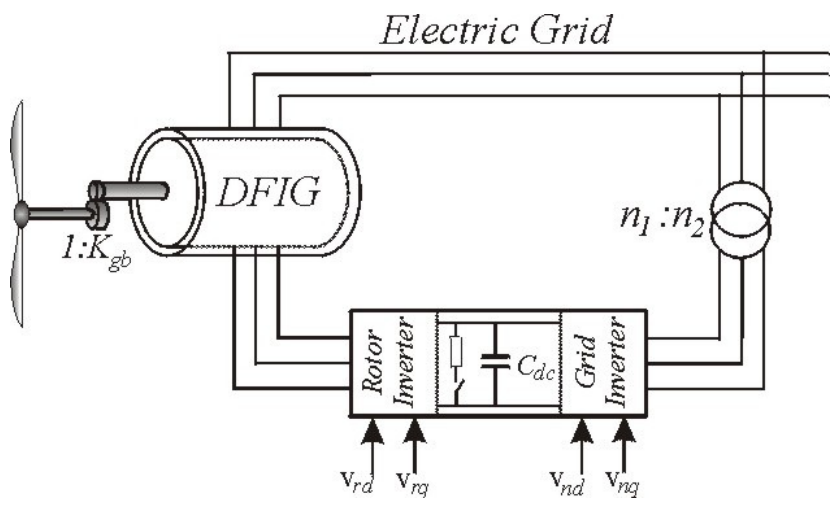

Fig. 1: DFIG-WECS structure

The dynamic model of this WECS is developed assuming the following assumptions: $i$ ) the magnetic flux into the air gap is sinusoidal distributed. Therefore, the analysis is made only for fundamental frequency components; $i$ ) a rigid shaft is considered; iii) the pitch angle is fix in the operational zone considered (active zone).

Using a synchronous reference frame, the dynamic equations corresponding to the turbine/DFIG set can be written as [4]:

$$
\begin{gathered}
\dot{\boldsymbol{\psi}}_{s}=-R_{s} \boldsymbol{I}_{\boldsymbol{s}}-j \omega_{e} \boldsymbol{\psi}_{s}+\boldsymbol{V}_{\boldsymbol{s}} \\
\dot{\boldsymbol{\psi}}_{r}=-R_{r} \boldsymbol{I}_{r}-j\left(\omega_{e}-\omega_{r}\right) \boldsymbol{\psi}_{r}+\boldsymbol{V}_{r} \\
\dot{\boldsymbol{\omega}}_{r}=\frac{P}{2 J}\left(T_{t}\left(\omega_{r}, v\right)-T_{e}\right)
\end{gathered}
$$

where $\boldsymbol{\psi}_{s}=L_{s} \boldsymbol{I}_{\boldsymbol{s}}+L_{m} \boldsymbol{I}_{r}$ and $\boldsymbol{\psi}_{r}=L_{r} \boldsymbol{I}_{r}+L_{m} \boldsymbol{I}_{s}$ are stator and rotor spatial flux vectors; $\boldsymbol{I}_{\boldsymbol{s}}$ and $\boldsymbol{I}_{r}$ stator and rotor spatial current vectors; $\omega_{e}$ is the electric frequency; $\omega_{r}$ is the rotational electric frequency; $\boldsymbol{V}_{\boldsymbol{s}}$ and $\boldsymbol{V}_{r}$ are spatial voltage vectors on stator and rotor terminals, respectively and $T_{e}$ is the electromagnetic torque. The parameters $R_{s}, R_{r}, L_{s}, L_{r}$ and $L_{m}$ correspond to stator and rotor winding resistances and inductances while $P$ is the total pole number and $J$ is the inertia of the rotational parts. Finally, $\mathrm{T}_{t}\left(\omega_{r}, v\right)$ represents the impeller torque generated by the wind turbine which is a function of the mechanical shaft speed $\left(\omega_{m}=2 K_{g b} \omega_{r} / P\right)$ and the wind speed $(v)$. This torque is related to the mechanical power of the turbine as:

$$
P_{t}\left(\omega_{r}, v\right)=T_{t} \omega_{m}=\frac{1}{2} \rho A C_{p} v^{3}
$$

where $\rho$ is the air density, $A$ the cross turbine area and $C_{p}$ is a wind speed and angular shaft speed dependent turbine coefficient which shows the turbine efficiency [10].

\section{A. Grid Balanced Conditions}

During grid balanced conditions the stator flux remains approximately constant. Neglecting the resistive voltage droop on stator windings, (1) can be expressed as:

$$
\boldsymbol{V}_{\boldsymbol{s}}=j \omega_{e} \boldsymbol{\psi}_{\mathrm{s}}
$$

On the other side, the stator current can be written as:

$$
\boldsymbol{I}_{\boldsymbol{s}}=\left(\boldsymbol{\psi}_{\mathrm{s}}-L_{m} \boldsymbol{I}_{r}\right) / L_{s}
$$

Replacing in (2), the quadrature components of the rotor dynamics result:

$$
\begin{aligned}
& i_{r d}=\frac{1}{\sigma L_{r}}\left(-R_{r} i_{r d}+\omega_{s l} i_{r q}+\frac{L_{m}}{L_{s}} \omega_{s l} \psi_{s q}+\mathrm{v}_{r d}\right) \\
& i_{r q}=\frac{1}{\sigma L_{r}}\left(-R_{r} i_{r q}+\omega_{s l} i_{r d}-\frac{L_{m}}{L_{s}} \omega_{s l} \psi_{s d}+\mathrm{v}_{r q}\right)
\end{aligned}
$$

where $\sigma=1-L_{m}^{2} / L_{s} L_{r}$ and $\omega_{s l}=\omega_{e}-\omega_{r}$.

Considering the synchronous frame aligned with the stator voltage, the reactive stator power can be calculated through the expression:

$$
\begin{gathered}
Q_{s}=\mathfrak{I} m\left(-\frac{3}{2} \boldsymbol{V}_{s} \times \boldsymbol{I}_{s}^{*}\right)= \\
=\frac{3}{2} \frac{\omega_{e}}{L_{s}}\left(\psi_{s d} L_{m} i_{r q}-\psi_{s q}\left(\psi_{s q}-L_{m} i_{r d}\right)\right) \cong \\
\cong-\frac{3}{2} \frac{\omega_{e}}{L_{s}} \psi_{s}\left(\psi_{s}-L_{m} i_{r d}\right)
\end{gathered}
$$

Analogously, the total electromagnetic power can be obtained as:

$$
P_{e}=-\frac{3}{2} \mathfrak{R} e\left(j \omega_{e} \boldsymbol{\psi}_{s} \times \boldsymbol{I}_{s}^{*}+j\left(\omega_{e}-\omega_{r}\right) \boldsymbol{\psi}_{r} \times \boldsymbol{I}_{r}^{*}\right)
$$

and therefore the electromagnetic torque results:

$$
T_{e}=\frac{3}{2} \frac{L_{m}}{L_{s}}\left(\psi_{s d} i_{r q}-\psi_{s q} i_{r d}\right) \cong \frac{3}{2} \frac{L_{m}}{L_{s}} \psi_{s} i_{r q}
$$

Given that the direct stator flux component can be practically neglected, from (9) and (11) it can be observed that the electromagnetic torque is essentially decoupled from the reactive stator power.

The DOIG rotor windings are linked to the grid through a B2B converter with a central DC bus and a chopper of resistance $R_{c h}$. The DC voltage on this bus can be expressed as:

$$
\dot{\mathrm{v}}_{d c}=\frac{1}{C_{d c}}\left(\frac{P_{r}-P_{n}}{\mathrm{v}_{d c}}-\frac{\mathrm{v}_{d c}}{R_{c h}} \delta\right)
$$

where $P_{r}=3\left(\mathrm{v}_{r d} i_{r d}+\mathrm{v}_{r q} i_{r q}\right) / 2, P_{n}=3\left(\mathrm{v}_{n d} i_{n d}+\mathrm{v}_{n q} i_{n q}\right) / 2$, and $i_{n d}$ and $i_{n q}$ are the synchronous direct and quadrature components of the current injected into the grid. On the other hand $\mathrm{v}_{n d}$ and $\mathrm{v}_{n q}$ are the direct and quadrature components respectively (imposed by the grid converter), and $\delta \in[0 ; 1]$ is the chopper switching function. 
Finally, the dynamic equations describing the interaction between the DC bus and the grid can be written as:

$$
\begin{gathered}
\dot{i}_{n d}=\frac{1}{L_{n}}\left(-R_{n} i_{n d}+\omega_{n} L_{n} i_{n q}+\mathrm{v}_{n d}-\mathrm{v}_{s}\right) \\
\dot{i}_{n q}=\frac{1}{L_{n}}\left(-R_{n} i_{n q}-\omega_{n} L_{n} i_{n d}+\mathrm{v}_{n q}\right)
\end{gathered}
$$

where $R_{n}$ and $L_{n}$ are the resistance and inductance of the Thevenin grid equivalent circuit, $\mathrm{v}_{\mathrm{s}}$ is the grid voltage and $\omega_{n}$ is the electric network frequency.

In this context, the active and reactive power injected into the grid through this converter can be expressed as:

$$
\begin{aligned}
& P_{n}=\frac{3}{2} \mathrm{v}_{s} i_{n d} \\
& Q_{n}=\frac{3}{2} \mathrm{v}_{s} i_{n q}
\end{aligned}
$$

From (15) and (16) it can be noted that selecting an appropriate synchronous rotating frame the power expressions explicitly show their decoupled behavior.

The complete WECS dynamic model is then described by (3), (9), (10), (12), (13) and (14) resulting in a multivariable and nonlinear complex system with five control inputs: $\mathrm{v}_{r d}, \mathrm{v}_{r q}, \mathrm{v}_{n d}, \mathrm{v}_{n q}$ and $\delta$.

\section{B. Grid Unbalanced Conditions}

Under grid voltage unbalances, the DOIG stator voltage can be considered composed by the conjunction of a positive and a negative three-phasic sequence. Seen from a stationary reference frame $\alpha-\beta$, this can be written as [3]:

$$
V_{\alpha \beta}=\left|V_{\alpha \beta+}\right| e^{j \omega_{e} t+\phi_{+}}+\left|V_{\alpha \beta-}\right| e^{-j \omega_{e} t+\phi_{-}}
$$

where the sub-indexes + and - refer to the considered sequence and $\square_{+}$and $\square_{-}$are the initial phase shifts which depend on the magnitude of the voltage unbalance and instant time where it ocurrs.

From a positive synchronous $d-q$ rotating frame, (17) can be rewritten as:

$$
V_{d q}^{+}=V_{\alpha \beta} e^{-j \omega_{e} t}=V_{d q+}^{+}+V_{d q_{-}}^{-} e^{-2 j \omega_{e} t}
$$

where the super-indexes + and - determine if the variable is seen from a positive or a negative synchronous reference frame, respectively.

Once the transitory produced by any unbalance is extinguished, the stator flux corresponding to each voltage sequence can be considered as constant $\left(R_{S} \approx 0\right)$. Therefore, from (1):

$$
\begin{aligned}
& V_{s d q}^{+}=j \omega_{e} \psi_{s d q}^{+}+\dot{\psi}_{s d q}^{+}=j \omega_{e}\left(\psi_{s d q+}^{+}+\psi_{s d q-}^{-} e^{-2 j \omega_{e} t}\right)+ \\
& +\frac{d\left(\psi_{s d q_{+}}^{+}+\psi_{s d q_{-}}^{-} e^{-2 j \omega_{e} t}\right)}{d t}=j \omega_{e}\left(\psi_{s d q_{+}}^{+}-\psi_{s d q_{-}}^{-} e^{-2 j \omega_{e} t}\right)
\end{aligned}
$$

On the other hand, from (7) the stator current can be expressed as:

$$
\begin{gathered}
I_{s d q}^{+}=\frac{1}{L_{s}}\left(\psi_{s d q}^{+}-L_{m} I_{r d q}^{+}\right)=\frac{1}{L_{s}}\left(\psi_{s d q+}^{+}+\psi_{s d q-}^{-} e^{-2 j \omega_{e} t}\right)- \\
-\frac{L_{m}}{L_{s}}\left(I_{r d q+}^{+}+I_{r d q-}^{-} e^{-2 j \omega_{e} t}\right)
\end{gathered}
$$

Thus, active and reactive stator powers can be calculated from:

$$
P_{s}+j Q_{s}=-\frac{3}{2} V_{s d q}^{+} \times I_{s d q}^{+*}
$$

and written in a compressed form as:

$$
\begin{gathered}
P_{s}=P_{s_{0}}+P_{s_{\mathrm{sin}}} \sin \left(2 \omega_{e} t\right)+P_{s_{\mathrm{cos}}} \cos \left(2 \omega_{e} t\right) \\
Q_{s}=Q_{s_{0}}+Q_{s_{\mathrm{sin}}} \sin \left(2 \omega_{e} t\right)+Q_{s_{\mathrm{cos}}} \cos \left(2 \omega_{e} t\right)
\end{gathered}
$$

where $P_{s_{0}}, P_{s_{\text {sin }}}, P_{s_{\text {cos }}}, Q_{s_{0}}, Q_{s_{\text {sin }}}$ and $Q_{s_{\text {cos }}}$ correspond to six terms additions of cross products between flux and current components of each sequence (see [4] for details). Eq. (22) and (23) show up that during grid voltage unbalances both active and reactive stator power present double frequency permanent oscillations. This effect can be also appreciated in the total electromagnetic power and therefore in the torque which can be calculated as:

$$
\begin{aligned}
T_{e}= & -\frac{3}{2} \mathfrak{R} e\left(j \omega_{e} \psi_{s d q}^{+} \times I_{s d q}^{+*}+j\left(\omega_{e}-\omega_{r}\right) \psi_{r d q}^{+} \times I_{r d q}^{+*}\right)= \\
& =\left(P_{e 0}+P_{e_{\sin }} \sin \left(2 \omega_{e} t\right)+P_{e_{\cos }} \cos \left(2 \omega_{e} t\right)\right) / \omega_{r}
\end{aligned}
$$

where analogously $P_{e 0}, \quad P_{e_{\mathrm{sin}}}$ and $P_{e_{\mathrm{cos}}}$ present expressions involving a six term addition of cross products between flux and current components of each sequence.

These undesirable oscillations under unbalanced grid conditions can produce stability problems on the grid and dangerous stress on the mechanical structure [3][4].

\section{Control System}

\section{A. Control Objectives}

To fulfill the recommendations of actual grid connection codes the main control objectives pursued in this work are the regulation of the total active and reactive power injected into the grid. Given that this WECS allows to independently regulate the grid power injection by stator and through the grid side converter, these active power sources should be coordinated not to produce deep excursions of some internal system variables like the DC bus voltage.

On the other hand, taking into account that during network unbalances it is desirable to reduce mechanical oscillations, the active power control objective is modified and indirectly fulfilled through the electromagnetic torque control. Under these directives and having in mind that the control design is carried out using Second Order Sliding Modes (SOSM) techniques, the control objectives are expressed in terms of the following set of sliding variables:

$$
\begin{gathered}
s_{1}=P_{r e f} / \omega_{r}-T_{e} \\
s_{2}=Q_{s_{r e f}}-Q_{s} \\
s_{3}=V_{d c_{r e f}}-\mathrm{v}_{d c} \\
s_{4}=Q_{n_{r e f}}-Q_{n}
\end{gathered}
$$

all of them having the required relative degree. 


\section{B. Control Design}

Let's consider a nonlinear multivariable dynamic system model under the structure:

$$
\dot{\boldsymbol{x}}=\boldsymbol{F}(\boldsymbol{x})+\boldsymbol{G} \boldsymbol{u}+\boldsymbol{\xi}(t)
$$

where $\boldsymbol{x} \in \mathfrak{R}^{n}$ represents the state vector and $\boldsymbol{u} \in \mathfrak{R}^{m}$ is the control vector, $\boldsymbol{F}$ represents the nominal drift vector, $\boldsymbol{G}$ is the control matrix and $\xi(t)$ represents the influence of bounded and continuous external perturbations.

Then, considering the sliding vector $\boldsymbol{S}=\left[\begin{array}{llll}S_{1} & S_{2} & S_{3} & S_{4}\end{array}\right]^{T}$ it is straightforward to obtain $\dot{\boldsymbol{S}}$ whose elements are given by [11]:

$$
\dot{s}_{i}=L_{\boldsymbol{F}} s_{i}+\sum_{j=1, j \neq i}^{m} L_{\boldsymbol{g}_{j}} s_{i} u_{j}+L_{\boldsymbol{g}_{i}} s_{i} u_{i}+\frac{\partial s_{i}}{\partial t}
$$

$i=1: 4$, where $L_{F} s_{i}, L_{g_{j}} s_{i}$ and $L_{g_{i}} s_{i} \neq 0$ correspond to Lie derivatives (i.e. $L_{\boldsymbol{F}} s_{i}=\frac{\partial s_{i}}{\partial \boldsymbol{x}^{T}} \boldsymbol{F}$ ) and $\boldsymbol{g}_{\mathrm{j}}$ denotes the $j$ th column of $\boldsymbol{G}$.

Once all the expressions involved in (30) have been calculated, sequentially taking equation $i$ from $m$ to 1 , obtaining $u_{i}$ and replacing it in the $i-1$ first equations, the system (3) is transformed into another one presenting a triangular control matrix where each line of it can be expressed as:

$$
\dot{s}_{i}=h_{f_{i}}(\boldsymbol{x}, \boldsymbol{\xi}, t)+\sum_{k=1}^{i} L_{g_{k}} s_{i} u_{k}
$$

Then, taking $i$ from 1 to $m$ there can be obtained $m$ SuperTwisting (STw) SOSM controllers:

$$
\begin{aligned}
& u_{i}=-\gamma_{s_{i}}\left|s_{i}\right|^{1 / 2} \operatorname{sgn}\left(s_{i}\right)+v_{i} \\
& \dot{v}_{i}=\left\{\begin{array}{cl}
-u_{i} & \left|u_{i}\right|>U_{M_{i}} \\
-\alpha_{s_{i}} \operatorname{sgn}\left(s_{i}\right) & \left|u_{i}\right| \leq U_{M_{i}}
\end{array}\right.
\end{aligned}
$$

which guarantee a stepped convergence of the system towards the manifold $s_{i}=\dot{s}_{i}=0$ in the order $i=m$ to 1 with finite reaching time. It should be noted that the convergence order is imposed by the analytic procedure followed, which in the step $i=k$ is considering that the system is already operating on the $m-k$ last surfaces.

To guarantee this convergence the controller parameters must fulfill the following inequalities [11][12]:

$$
\begin{gathered}
\frac{K_{s_{i}}}{\alpha_{s_{i}}}>C_{s_{i}} \\
\gamma_{s_{i}}>\sqrt{\frac{2}{\underline{K}_{s_{i}} \alpha_{s_{i}}-C_{s_{i}}}} \frac{\bar{K}_{s_{i}}\left(\underline{K}_{s_{i}} \alpha_{s_{i}}-C_{s_{i}}\right)\left(1-q_{s_{i}}\right)}{\bar{K}_{s_{i}}^{2}\left(1+q_{s_{i}}\right)}
\end{gathered}
$$

where:

$$
\begin{gathered}
0<q_{s_{i}}<1 \\
0<\underline{K}_{s_{i}}<L_{g_{i}} s_{i}<\bar{K}_{s_{i}}
\end{gathered}
$$

$$
\begin{aligned}
& \left|L_{(\boldsymbol{F}+\boldsymbol{\xi})} h_{f_{i}}+\frac{\partial h_{f_{i}}}{\partial t}\right|+\sum_{k=1}^{m}\left|L_{\boldsymbol{g}_{k}} h_{f_{i}}\right| U_{M_{k}}+ \\
& +\sum_{k=1}^{i-1}\left|L_{g_{k}} s_{i}\right| \overline{\dot{u}}_{k} \leq C_{s_{i}} \\
& \left|\frac{h_{f_{i}}(\boldsymbol{x}, \boldsymbol{\xi}, t)+\sum_{k=1}^{i-1} L_{g_{k}} s_{i} U_{M_{k}}}{L_{g_{i}} s_{i}}\right|<q_{s_{i}} U_{M_{i}}
\end{aligned}
$$

inside the operational system limits and the bounded perturbations and uncertainties considered. The controllers obtained in this way determine that the second time derivative of each surface belongs to the differential inclusion:

$$
\ddot{s}_{i} \in\left[\begin{array}{ll}
-C_{s_{i}} & C_{s_{i}}
\end{array}\right]+\left[\begin{array}{ll}
\underline{K}_{s_{i}} & \bar{K}_{s_{i}}
\end{array}\right] v_{i}
$$

This inclusion contains the nominal system dynamics and all its possible deviations produced by the set of bounded perturbations and parameter variations acting on the system and considered during the design tasks.

The described methodology implies to follow an extended algebraic procedure to finally obtain the controller parameters which typically determine very conservative controllers. Then, after that first analytic step these parameters are commonly adjusted using complementary tools like computer simulations. This tuning task is carried out only once during the controller design.

Following these guidelines and considering the WECS parameters shown in the Appendix, the multivariable STw-SOSM controller is given by:

$$
\begin{array}{llll}
\alpha_{s 1}=-950 & \alpha_{s 2}=-750 & \alpha_{s 3}=-150 & \alpha_{s 4}=540 \\
\gamma_{s 1}=-0.014 & \gamma_{s 2}=-0.015 & \gamma_{s 3}=-4 & \gamma_{s 4}=1.5 \\
\text { C. Unbalanced Conditions } &
\end{array}
$$

Despite STw-SOSM controllers are robust against the parametric variations and bounded external perturbations considered during its design, they are not able to completely reject the kind of disturbances produced by grid unbalances. These double grid frequency oscillations on active/reactive powers, currents and electromagnetic torque are very dangerous for the network stability and the mechanical structure and according to the wind energy connection grid codes they must be mitigated.

In order to fulfill this control demand during the last years researchers have been following two possible lines. One of them is based on voltage/current sequence decomposition [4][5][6][7]. The other proposes a combination of controllers with resonant filters [3]. The control proposal of this work is based on this second alternative which determines simpler and reduced control topologies avoiding errors and delays in the sequence calculus.

To endorse the control system with such a capability each STw-SOSM loop is combined (see Fig.1) with two resonant filters in parallel which introduce a high gain loop value on the particular frequencies $\omega_{e}$ and $2 \omega_{e}$. Once these filters are correctly tuned in coordination with the STw-SOSM controllers, the overall system presents an 
important reduction on the power and torque oscillations levels. The tuning procedure determined the following parameters for the resonant filters used:

$$
F R_{1}(s)=\frac{k_{1_{i}} s}{s^{2}+k_{2_{i}} s+\omega_{e}^{2}} ; F R_{2}(s)=\frac{k_{3_{i}} s}{s^{2}+k_{4_{i}} s+\left(2 \omega_{e}\right)^{2}}
$$

\begin{tabular}{|c|c|c|c|c|}
\hline & $k_{1 i}$ & $k_{2 i}$ & $k_{3 i}$ & $k_{4 i}$ \\
\hline$s_{1}$ & 200 & 9.42 & 500 & 1.57 \\
\hline$s_{2}$ & 40 & 9.42 & 100 & 1.57 \\
\hline$s_{3}$ & 25 & 4.71 & 350 & 15.7 \\
\hline$s_{4}$ & 250 & 4.71 & 350 & 15.7 \\
\hline
\end{tabular}

To complement this design and reduce the voltage oscillations on the DC bus the control scheme is completed with the action of a resistive chopper which is operated under a simple switching control law like:

$$
\delta= \begin{cases}1 & \mathrm{v}_{\mathrm{dc}}>V_{d c_{\text {rated }}} \\ 0 & \mathrm{v}_{\mathrm{dc}} \leq V_{d c_{\text {rated }}}\end{cases}
$$

implemented with an associated hysteresis cycle not to produce a continuous switching behavior. The hysteresis width is adjusted according to the admissible ripple on $\mathrm{v}_{d c}$.

\section{Simulation Results}

This section presents representative simulation results which permits analyze the overall control system performance under normal and unbalanced grid conditions. The system parameters are specified in the Appendix.

\section{A. Balanced Conditions}

Under balanced grid conditions the WECS operation is simulated considering a variable wind speed (below the rated wind speed) and assuming stepped active and reactive power references hypothetically determined by the grid operator. It should be noted that these power references are selected considering the limit imposed by the wind conditions (total energy availability). The time profiles of all these variables are depicted in Fig.3.
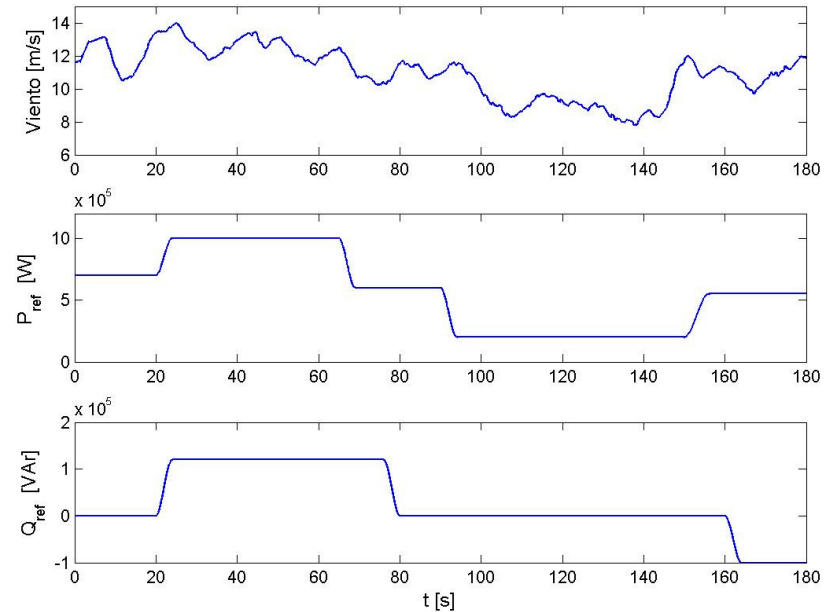

Fig. 3: (a) Wind speed; (b) Total active power reference; (c) Stator reactive power reference

The system behavior can be analyzed through the sliding variables time profiles shown in Fig.4. These graphs corroborate that the multivariable STw-SOSM controller is capable to efficiently and simultaneously follow the four sliding surfaces previously defined, presenting a low ripple level determined by the switching frequency used on the static converters $(5 \mathrm{KHz})$. The abrupt excursion observed on Fig.4c and Fig.4d at $t=2 \mathrm{~s}$ corresponds to the reaching transitory of these sliding variables produced by an on purpose delayed start of these controllers.

As it was previously specified, the generated active power is injected into the network through the stator windings and the grid side inverter. To simultaneously regulate the system on the power level required and deal with mechanical perturbations, it is convenient to transform the active power reference (given by the grid operator) into an electromagnetic torque reference. In this way resonant filters to mitigate dangerous oscillations can be directly included into this 'mechanical' loop. The active power injected through the grid side converter is indirectly controlled by regulating the DC-bus voltage on its rated value.
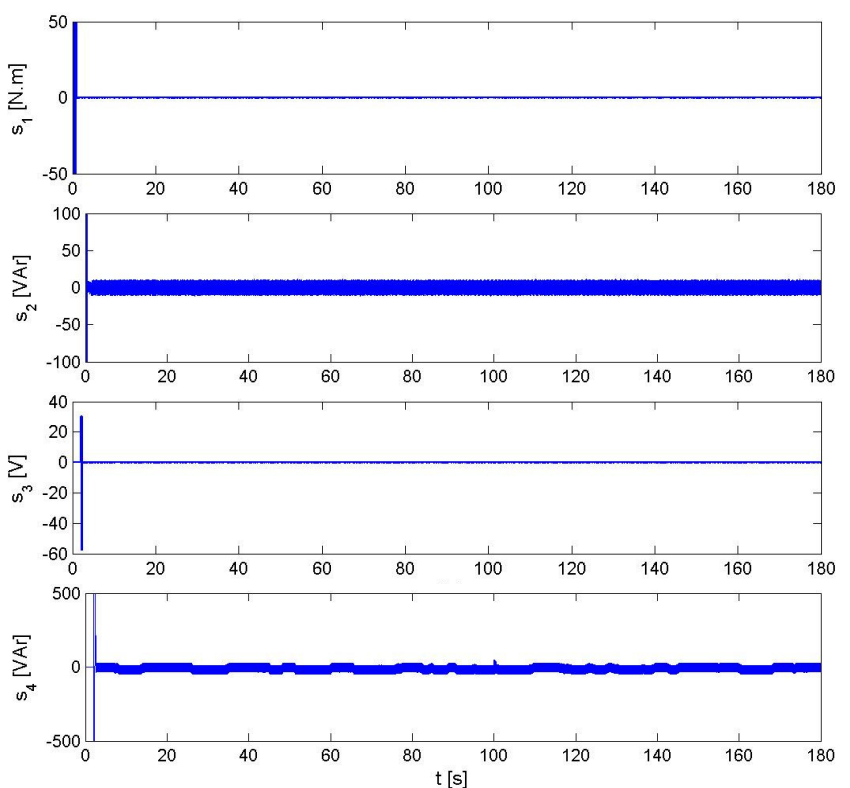

Fig. 4: (a) $s_{1}=T_{r e f}(t)-T_{e}$; (b) $s_{2}=Q_{r e f}-Q_{s}$; (c) $s_{3}=V_{d c}$ ref $-v d c$; (d) $s_{4}=Q_{n \text { ref }}-Q_{n}$

\section{B. Unbalanced Conditions}

To recreate abnormal grid conditions, two consecutive and sudden unbalances were considered. In particular $10 \%$ monophasic voltage falls from $t=1 \mathrm{sec}$. to $t=3 \mathrm{sec}$. and $t=5.5 \mathrm{sec}$. to $t=8 \mathrm{sec}$. were included in the simulations.

The system performance is shown through the sliding variables behavior in Fig.5. In this figure it can be seen that the system present transitory excursions outside the sliding surfaces when the voltage unbalance appear and when it disappear. However due to the complementary action of resonant filters and the STw-HOSM controller the upper bounds of these excursions are not very important. It should be emphasized that the control design was focalized on the oscillation level reduction and therefore the transitory duration requirements were relaxed.

It is interesting to note that once the transitory response is extinguished the oscillations levels are very low. When normal grid conditions are recovered the control system 
tries to restore the sliding motion. However, this process results a bit slow in some of the surfaces (especially on $s_{1}$ and $s_{2}$ ) because the corresponding resonant filters maintains on their outputs $50 \mathrm{~Hz}$ sustained oscillations. This behavior is being under study and it is matter of future work.
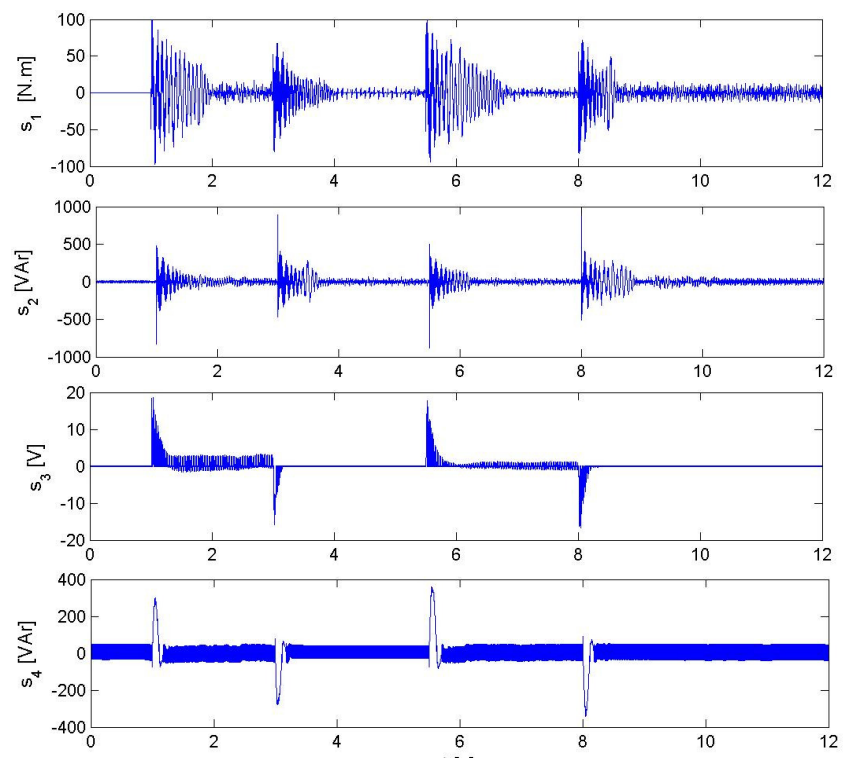

Fig. 5: (a) $s_{1}=T_{r e f}(t)-T_{e}$; (b) $s_{2}=Q_{r e f}-Q_{s}$; (c) $s_{3}=V_{d c} r e f-v d c$; (d) $s_{4}=Q_{n \text { ref }}-Q_{n}$

\section{Conclusion}

In this article a multivariable nonlinear power control applied to a DOIG-WECS linked to the utility grid was presented. The control technique (STw-SOSM) used to develop the current design naturally incorporates the nonlinear nature of the system model, its parametric uncertainties and external perturbations, presenting good robustness features and a successful performance in the variable references tracking (stepped power references) under variable wind speed profiles. The resultant control law is simple to implement presenting a computational burden not bigger than the one to run a PID controller and not depends on system parameters.

To endorse the control system with the capability of mitigate the effects produced by network unbalances, the multivariable controller was complemented with resonant filters which are appropriately tuned on specific frequencies. The simulation tests carried out using representative situations show that the presented control scheme successfully deals with this kind of anomaly attaining a good oscillation reduction in the active/reactive power injected into the net and also on the mechanical structure. This feature allows reducing harmful effects on the grid stability and the mechanical system structure.

\section{Appendix}

\begin{tabular}{|l|c|}
\hline DFIG-WECS Parameters & \\
\hline Rated Power & $2 \mathrm{MW}$ \\
\hline Line Voltage & $690 \mathrm{~V}$ \\
\hline$f_{n}$ & $50 \mathrm{~Hz}$ \\
\hline$R_{s}$ & $0.01 \mathrm{p} . \mathrm{u}$ \\
\hline$R_{r}$ & $0.009 \mathrm{p} . \mathrm{u}$ \\
\hline$L_{l s}$ & $0.171 \mathrm{p} . \mathrm{u}$ \\
\hline
\end{tabular}

\begin{tabular}{|l|c|}
\hline$L_{l r}$ & $0.156 \mathrm{p} . \mathrm{u}$ \\
\hline$L_{m}$ & $3.9 \mathrm{p} . \mathrm{u}$ \\
\hline$J$ & $331.93 \mathrm{Kg} \cdot \mathrm{m}^{2}$ \\
\hline$P$ & 6 \\
\hline$V_{D C}$ & $1200 \mathrm{~V}$ \\
\hline$R_{n}$ & $0.16 \Omega$ \\
\hline$L_{n}$ & $2.8 \mathrm{mHy}$ \\
\hline$C_{d c}$ & $20000 \mu \mathrm{F}$ \\
\hline
\end{tabular}

\section{References}

[1] A. Hansen and Michalke, "Multi-pole permanent magnet synchronous generator wind turbines' grid support capability in uninterrupted operation during grid faults". IET Renewable Power Generation, Vol. 3(3), pp. 333-348, 2008.

[2] A. Hansen and Michalke, "Modelling and control of variable-speed multi-pole permanent magnet synchronous generator wind turbine". Wind Energy, Vol. 11(5), pp. 537-554, 2008.

[3] P. Zhou, Y. He and D. Sun, "Improved direct power control of a dfig-based wind turbine during network unbalance", IEEE Trans. Power Electronics, Vol. 24(11), pp. 2465-2474, 2009.

[4] L. Xu and Y. Wang, "Dynamic modelling and control of dfig-based wind turbines under unbalanced network conditions", IEEE Trans. Power Systems, Vol. 22(1), pp. 314323, 2007.

[5] R. Zhu, Z. Chen, X. Wu and H. Liu, "High order sliding mode control of doubly-fed induction generator under unbalanced grid faults", IECON 2013 - 39th Annual Conference of the IEEE, Vienna, 2013.

[6] O. Gomis-Bellmunt, A. Junyent-Ferré, A. Sumper and J. Bergas-Jané, "Ride-Through Control of a Doubly Fed Induction Generator Under Unbalanced Voltage Sags", IEEE Transactions on Energy Conversion, vol. 23, no. 4, pp. 10361045, Dec. 2008

[7] Sol-Bin Lee and Kyo-Beum Lee, "Performance improvement of a DFIG in a wind turbine under an unbalanced grid-voltage condition", In: Proceedings of IEEE Int. Symp. on Ind. Elect., Bari, 2010.

[8] G. Bartolini, A. Pisano, E. Punta and E. Usai, "A survey of applications of second order sliding mode control to mechanical systems", Int. J. of Control, Vol. 76(9/10), pp. 875-892, 2003.

[9] A. Levant, "Introduction to high-order sliding modes", Available: http://www.tau.ac.il/ levant/hosm2002.pdf, 2003.

[10] Rolan, A., A. Luna, G. Vazquez, D. Aguilar and G. Azevedo, "Modeling of a variable speed wind turbine with a permanent magnet synchronous generator". In: Proceedings of IEEE Int. Symp. on Ind. Elect., 2009.

[11] Valenciaga, F. and D. Fernandez (2015). Mimo high order sliding mode control for a pmsg wind based system with grid support capabilities. IEEE Trans. Ren. Power Gen. 9(8), 925934.

[12] Levant, A. (2003b). Mimo 2-sliding control design. In: European Control Conf.. Cambridge, UK. 